\title{
Paediatric Chronic Myeloid Leukaemia in a Tertiary Hospital in Nigeria: A Report of Two Cases
}

Udechukwu Ngozi Patricia $^{1}$, Nwagha Theresa Ukamaka ${ }^{2}$, Okike Clifford ${ }^{1}$, Ukoh Uchechukwu Chukwuebuka ${ }^{1}$ and Oyim-Elechi Oluchi Cecilia ${ }^{1}$

1. Department of Paediatrics, Alex Ekwueme Federal University Teaching Hospital, Abakaliki 043, Ebonyi State, Nigeria

2. Department of Haematology \& Immunology, University of Nigeria Teaching Hospital, Ituku-Ozalla, PMB 01129, Enugu, Nigeria

\begin{abstract}
Background: Chronic myeloid leukaemia (CML) is a myeloproliferative disorder with BCR/ABL mutation characterized by proliferation of granulocytic cell lines at varying stages of development. It is predominantly a disease of the adult population, only accounting for 2-3\% of all childhood leukaemia. In our tertiary hospital, we recorded two cases of paediatric CML within three years. Objective: To increase the awareness of physicians on this seemingly rare form of childhood leukaemia. Case reports: We report a 10 year-old boy and a 16 year-old girl who presented to our facility with features suggestive of CML. Both cases had splenomegaly and leucocytosis. Cytogenetic assessment was positive for BCR-ABL in both cases. The first case was in chronic phase (CP) and had an initial therapy of hydroxyurea (HU) before referral for imatinib therapy. The second case received imatinib therapy, however due to blastic transformation to acute myeloid leukaemia (AML), she received treatment comprising of intravenous anthracycline and cytarabine. Conclusion: CML, although one of the rare childhood leukaemia is becoming increasingly noticed, probably due to availability of diagnostic facilities. There is therefore need for increased awareness of the disease among physicians to minimize delays in the diagnosis for improved care and better outcome.
\end{abstract}

Key words: Paediatric, CML, blastic transformation, imatinib.

\section{Introduction}

Chronic myeloid leukaemia (CML) is a rare childhood malignancy and accounts for $2-3 \%$ of all childhood leukaemias [1, 2]. It predominantly affects the adult population [3]. It is of two subtypes; adult-type CML which is Philadelphia-chromosome positive and the juvenile-type CML, which is Philadelphia-chromosome negative [4]. Paediatric CML accounts for less than $10 \%$ of all cases of CML [5].

It is one of the few malignancies known to be caused by a specific genetic mutation [3]. Its pathogenesis involves the mutual translocation of the Abelson murine leukaemia (ABL1) gene on chromosome 9, and its fusion with the breakpoint cluster region (BCR) gene on chromosome 22 , $t(9 ; 22)(q 34 ; 11)$, to form an

Corresponding author: Udechukwu Ngozi Patricia, FWACP, MB, BS, research fields: paediatric haematology, oncology. aberrant chromosome 22 which harbours an oncoprotein BCR-ABL1 [2, 3, 6, 7]. This new chromosome 22 is known as Philadelphia chromosome [8]. The BCR-ABL1 has tyrosine kinase activity that promotes growth and replication, and influences leukaemogenesis [6].

CML is an insidious disease with initial non-specific symptoms, which includes weight loss, asthenia, low grade fever, sweats, malaise and hepatomegaly [2, 7]. Splenomegaly is the most common sign seen in over $70 \%$ of the cases [1]. The disease has three phases; chronic phase (CP), accelerated phase (AP) and blast phase (BP) [2, 3, 6, 9]. Over $90 \%$ of patients are diagnosed in CP [1, 6]. The percentage of blast cells found in the bone marrow or peripheral blood film is used to categorize the phase of the disease, as well as the severity of the symptoms [10]. The blast phase of CML presents like the acute myeloid form in $60 \%$ of cases, and the lymphoid form in $30 \%$ of cases [6]. 
The diagnosis of CML is made based on the presence of the clinical features, marked leucocytosis with myeloid cells at all stages of differentiation [2]. It is confirmed with the demonstration of the Philadelphia chromosome $\mathrm{t}(9 ; 22)(\mathrm{q} 34 ; 11)$ and BCR-ABL transcripts [2, 6, 7, 11]. Philadelphia chromosome is detected using cytogenetic studies, which can be done using chromosome banding analysis or fluorescence in-situ hybridization (FISH) technique $[6,7]$. The BCR-ABL transcripts are detected using reverse transcript polymerase chain reaction (RT-PCR) [7]. Qualitative RT-PCR gives information on the BCR-ABL transcript type, while quantitative RT-PCR gives information on the number of transcripts and can be used to monitor the progress of the disease during treatment.

Historically, treatment of CML has progressed from the use of Busulfan, to HUs and Interferon- $\alpha$ (INF $\alpha$ ) over the years, and now tyrosine kinase inhibitor (TKI) is the gold standard [7]. Imatinib mesylate is the first TKI introduced, and is still the first line drug of choice for treatment of CML in chronic phase in adults and children [8]. Other TKIs approved for the treatment of CML include dasatinib and nilotinib. TKIs act through competitive inhibition at the ATP-binding site of the BCR-ABL1 oncoprotein, which results in the inhibition of phosphorylation of proteins involved in cell signal transduction [6]. Haematopoietic stem cell transplant (HSCT) is currently the only curative treatment option available for CML management in both adults and children. The rarity of CML in children has led to fewer studies describing the clinical features or pattern of presentation and clinical practice protocols unlike the case of adult CML [8]. The non-specificity of clinical features causes delays in the diagnosis. The two cases are therefore reported to increase the index of clinical suspicion of physicians on this rare form of childhood leukaemia.

\section{Methodology}

We report two cases of paediatric CML seen and managed in the paediatric haemato-oncology unit of a tertiary hospital, Alex-Ekwueme Federal University Teaching Hospital Abakaliki (AE-FUTHA) between March 2013 and July 2016.

Case 1 is a 10-year-old boy who was presented in March 2013, with complaints of abdominal swelling and recurrent fever of two months duration. Physical examination revealed pallor, there was no significant peripheral lymph adenopathy. There was hepatomegaly of $8 \mathrm{~cm}$ and splenomegaly $18 \mathrm{~cm}$ below the costal margins. Full blood count (FBC) revealed packed cell volume (PCV) of 28\%, leucocytosis of 383,000 cells $/ \mathrm{mm}^{3}$. Differentials were as follows: neutrophils: $85 \%$, lymphocytes: $6 \%$, Eosinophil: $4 \%$, monocytes: $2 \%$ and basophils: $3 \%$; platelets were $412 \times$ $10^{9} / \mathrm{L}$. Peripheral blood film and bone marrow smears showed hyper cellular marrow fragments with leucocytosis and sequential maturation of myeloid cell line; myelocytes and promyelocytes. Cytogenetic study was positive for Philadelphia chromosome (BCR-ABL) confirming the diagnosis of CML, BCR-ABL1 transcript type: e13a/e13a2. BCR-ABL ratio: 42.042\%. Serum electrolytes, urea and creatinine, liver enzymes and serum uric acid were within normal ranges.

Following two weeks of no appreciable clinical and haematological response with treatment on $\mathrm{HU}$, he was referred to Obafemi Awolowo University Teaching Hospital (OAUTH) Ile-Ife where he was enrolled into Glivec International Patient Assistance Programme (GIPAP) [10] for imatinib therapy. This patient however was lost to follow up following this referral.

Case 2 is a 16-year-old female, who presented in July 2016, with a history of recurrent fever and abdominal distension of four weeks duration. The abdominal distension was associated with discomfort and occasional non-projectile vomiting. She had been hospitalized twice in the past 13 months at which times she was managed for sepsis and then severe malaria.

Examination revealed an ill-looking, febrile, moderately pale, asthenic built adolescent with no significant peripheral lymphadenopathy. The liver and 
spleen were $13 \mathrm{~cm}$ and $22 \mathrm{~cm}$ enlarged below the costal margins.

FBC showed severe leucocytosis of 522,000 cells $/ \mathrm{mm}^{3}$, neutrophilia of $89 \%$ with relative lymphopenia of $3 \%$. Blood film showed marked leucocytosis, with distinct myeloid precursor cells, transverse maturation of myeloid cell line, few blasts $(<5 \%)$, and decreased number of platelets. Bone marrow smear revealed hypercellularity with increased myeloid precursor cells with blasts $<5 \%$. Cytogenetic assessment was positive for BCR-ABL confirming the diagnosis of CML in CP, BCR-ABL1 transcript type: e14a2, BCR-ABL ratio: $14.52 \%$. She was subsequently referred to OAUTH Ile-Ife where she received HU for two weeks before being commenced on imatinib therapy in the GIPAP to be monitored as an outpatient in AEFUTHA since they live in Abakaliki, but they did not come. However, due to disease progression to blast transformation she was referred back to AEFUTHA three months later. She re-presented at our facility with generalized body pains, severe headache, refusal to eat, mouth sores and tiredness on mild exertion. Aside referral letter, they also came with FBC result done immediately after two weeks of HU therapy showing PCV of $31 \%$ and total WBC of $7.4 \times 10^{9} / \mathrm{L}$.

She was severely pale, febrile $\left(39.4^{\circ} \mathrm{C}\right)$, had a pulse rate of 136 beats per minute which was bounding and was also in respiratory distress (nasal flaring and tachypnoea of 40 breaths per minute), though the chest was clear to auscultation. Other findings were angular stomatitis, purpuric skin rash, lower motor neuron type right facial nerve palsy; and splenomegaly of $16 \mathrm{~cm}$ and tender hepatomegaly of $8 \mathrm{~cm}$ below the costal margins.

A diagnosis of sepsis with CNS involvement in a child with CML (blast phase) was made. Available investigation results showed serum uric acid, liver function test, serum electrolytes, urea and creatinine were within normal limits. Cerebrospinal fluid cytology showed no malignant cells. FBC showed PCV of $17 \%$, total white blood cell (WBC) count was $2.9 \times$
$10^{9} / \mathrm{L}$ with absolute neutrophil count of 580 cells $/ \mathrm{mm}^{3}$; platelet was $26.0 \times 10^{9} / \mathrm{L}$. Electrocardiography showed evidence of left ventricular hypertrophy, left axis deviation and prolong QT interval.

She was resuscitated with antibiotics, blood component therapy and other supportive measures which resulted in clinical improvement. Due to cost of treatment (funded from out of pocket expenditure) there was a delay of four weeks before commencement of chemotherapy. She was subsequently commenced on actinomycin D, 6-mecaptopurine and intrathecal methotrexate as parents could not afford cytarabine. With financial support from a state government program, she was then able to receive the full regimen with cytarabine inclusive, she got two cycles of this regimen. Her condition improved subsequently and the last FBC before expiration of government sponsorship by December 31, 2017 is as follows: - PCV: 29\%, total WBC: $49.7 \times 10^{9} / \mathrm{L}$; Differentials were neutrophils: $68 \%$, lymphocytes: $10 \%$, eosinophils: $6 \%$, monocytes: 5\% and basophils: $3 \%$; blasts were $8 \%$ and platelet count was $371.0 \times 10^{9}$, a picture close to CP of CML. She remained clinically stable though not in full remission. Two months after expiration of financial support, she was readmitted for disease relapse resulting from discontinuation of chemotherapy and she succumbed to her illness.

\section{Discussion}

We reported two cases of Philadelphia chromosome-positive CML out of 10 cases of leukaemia, and 51 cases of childhood malignancies seen over the same period in this institution. This gives a prevalence of $3.9 \%$. This is similar to the prevalence of 3.4\% reported by Oyekunle et al. [4] but higher than the prevalence of $1.9 \%$ and $0 \%$ reported by Chukwu et al. [12] and Babatunde et al. respectively [13]. The higher prevalence may be due to improved diagnostic capabilities, including capacity to do bone marrow and molecular studies. This implies that cases that would have gone undetected are now diagnosed and 
awareness is increased.

The two cases seen in our centre were aged 10 years and above. This is similar to previous reports by Ngolet et al [14] in Congo where all and Lalit Rau et al [15] in India where majority (77\%) of the CML patients were in the age range of 10-17 years. This supports the fact that CML is a disease more prevalent in adolescence. This underscores the need for a high index of suspicion in this age group in order to make early diagnosis with subsequent early intervention and better outcome.

Both patients had splenomegaly which is the most prominent clinical feature of the disease, similar to that reported by Millot et al [1] and Belgaumi et al [16]. In as much as splenomegaly can occur in many other tropical diseases and as such is a non-specific indicator, its persistent presence in an adolescent should raise suspicion to investigate for CML more so as this disease is already noted to be common in this age group.

Despite their late presentation, both cases were diagnosed in the CP just as reported by other centres $[12,15]$. This may be because of the usual long duration of the CP lasting three to four years. Despite the non-specific presentation at this phase, the duration gives the physician time to unravel the diagnosis.

Case 1 was managed with HU for two weeks but this did not give appreciable positive clinical and haematological response. This was probably due to poor efficacy of drugs obtained from a questionable source; the caregivers had procured the drugs by themselves from unregulated drug outlets which are prevalent in the environment. This contrasts with the response from case 2, in whom initial HU (from OAUTH Ile-Ife) therapy resulted in reduction in total WBC from above $500 \times 10^{9} / \mathrm{L}$ to $7.4 \times 10^{9} / \mathrm{L}$ and also as noted by previous researchers [7, 15]. However, commencement of imatinib therapy did not yield good response in this patient, similar to earlier reports by Ngolet et al [14] but differed from reports of Oyekunle et al [4], who reported that $93 \%$ of his study population achieved complete haematological response after 90-day therapy on imatinib, while two out of the 14 cases had complete cytogenetic remission. This poor response of our patient could be attributed to the poor prognostic factors seen in our patient, which included older age, massive splenomegaly, hepatomegaly, late presentation and Philadelphia chromosome positivity. Also case 2 had neutropenia, thrombocytopenia, and generalized body pains especially on the legs while on imatinib therapy; this could be as a result of imatinib toxicity similar to reports of Raut et al. [15] and Millot et al [17]. The prolonged QT interval and other cardiac abnormalities noted in case 2 may be due to imatinib toxicity as reported by previous researchers Kekela et al [18] and Andolina [19]. Kelela et al. reported for adults and gave findings of cardiac toxicity in their subjects; our patient is 16 years old and as such almost an adult and probably responded to imatinib like an adult. Andolina et al [19] in their review noted cardiotoxicity and growth retardation to be significant side effects.

Some centres reported more of pre-diagnostic delays while others reported more of post-diagnostic delays $[20,21]$. But in our centre, both types of delays punctuated our patients' management as demonstrated in case 2 . From the time of haematological diagnosis in AEFUTHA, it took up to seven months to confirm the diagnosis in a neighbouring city (Enugu) and another two and half months to commence treatment at OAUTH Ile-Ife. These delays were due to not having all the facilities in one place. The same also caused drug related toxicities; as initial HU treatment was not monitored due to distance between Abakaliki and Ile-Ife which is about $527 \mathrm{~km}$. The non-compliance to medication and instructions also played a role in these complications as the child was not presented to AEFUTHA for follow-up and monitoring despite advice to do so. This demonstrates the need for adequate counseling to ensure adherence to regular medications and adequate arrangements for monitoring before commencement of imatinib received from the GIPAP. 
The financial burden of cancer management on the patients and families in resource poor settings like ours is much, as the national health insurance scheme (NHIS) does not cover for oncology treatment and most of their health service payments were out of pocket. This contributed to treatment and monitoring delays and gaps until some sponsorship was obtained from the state government for the second case. Chemotherapy was not continued because this support lapsed on December 31, 2017; this led to the demise of the patient two months later. This underscores the need for robust sponsorship programs for the management of oncology cases; such programs as funded by philanthropist or grants or non-governmental organizations (NGOs).

\section{Conclusions}

CML, although one of the rare childhood leukaemias is becoming increasingly noticed, probably due to improved diagnostic capabilities like the GIPAP which is currently situated in OAUTH Ile-Ife in Southern Nigeria. There is therefore need for increased index of clinical suspicion among physicians to minimize delays in the diagnosis. This will lead to early diagnosis, prompt commencement of treatment, and overall improvement in survival outcome of patients. Such diagnostic facilities should be represented across the nation in order to increase access.

\section{Acknowledgements}

The authors are grateful to the other members of the team who took part in the management of the cases.

\section{References}

[1] Millot, F., Traore, P., Guilhot, J., Nelken, B., Leblanc, T., Leverger, G., et al. 2005. "Clinical and Biological Features at Diagnosis in 40 Children with Chronic Myeloid Leukemia.” Pediatrics 116 (1): 140-3.

[2] Tuberger, D. G., Bleyer, A., and Ritchey, A. K. 2011. Nelson Textbook of Paediatrics. 19th ed., editted by Kliegman, R. M., Stanton, B. F., Gene, J. W. S., Schor, N. F., and Bechrman, R. E. Philadelphia: Elsevier Saunders.

[3] Besa, E. C. 2018. “Chronic Myelogenous Leukaemia.”
Accessed Jan. $\quad 3$ 3, 2018. http://emedicine.medscape.com/article/199425.

[4] Oyekunle, A., Bolarinwa, R. A., Mamman, A. I., and Durosinmi, M. 2012. "The Treatment of Childhood and Adolescent Chronic Myeloid Leukaemia in Nigeria.” $J$ Pediatr Sci. 4 (4): 169.

[5] Brodeur, G. M., Dow, L. W., and Williams, D. L. 1979. "Cytogenetic Features of Juvenile Chronic Myelogenous Leukaemia.” Blood J. 53 (5): 812-9.

[6] Jabbour, E., and Kantarjian, H. 2016. “Chronic Myeloid Leukemia: 2016 Update on Diagnosis, Therapy, and Monitoring.” Am J Haematol. 91 (2): 253-65.

[7] Baccarani, M., Pileri, S., Steegmann, J. L., Muller, M., Soverini, S., and Dreyling, M. 2012. "Chronic Myeloid Leukemia: ESMO Clinical Practice Clinical Practice Guidelines for Treatment and Follow Up.” Ann Oncol. 23: vii72-7.

[8] Hijiya, N., Schultz, K. R., Metzler, M., Millot, F., and Suttorp, M. 2016. "Pediatric Chronic Myeloid Leukemia Is a Unique Disease That Requires a Different Approach.” Blood J. 127 (4): 392-400.

[9] Arber, D. A., Orazi, A., Hasserjian, R., Borowitz, M. J., Le Beau, M. M., Bloomfield, C. D., et al. 2016. “The 2016 Revision to the World Health Organization Classification of Myeloid Neoplasms and Acute Leukemia.” Blood J. 127 (20): 2391-406.

[10] Oyekunle, A., Durosinmi, M., Bolarinwa, R., Owojuyigbe, T., Salawu, L., and Akinola, N. 2016. "Chronic Myeloid Leukaemia in Nigerian Patients: Anemia Is an Independent Predictor of Overall Survival.” Clin Med Insights Blood Disord. 9: 9-13.

[11] Nowell, P. C. 2007. "Discovery of the Philadelphia Chromosome: A Personal Perspective.” J Clin Invest. 117 (8): 2033-5.

[12] Chukwu, B. F., Ezenwosu, O. U., Ikefuna, A. N., and Emodi, I. J. 2015. "A Prospective Study of Childhood Malignancy in Enugu, Eastern Nigeria (2011-2013).” Niger J Paediatr. 42 (2): 116-20.

[13] Babatunde, T., Ogun, G., Brown, B., Akang, E., and Aken, O. Y. A. 2014. "Pattern of Childhood Leukaemia in University College Hospital, Ibadan.” Afr J Med Med Sci. 43 (2): 135-8.

[14] Ngolet, L. O., Okouango, J. G. O., and Dolekia, A. E. 2017. "CML in a Paediatric Population in Congo." Med Sci Discov. 4 (3): 27-9.

[15] Raut, L., Bohara, V. V., Ray, S. S., Chauchuri, P., and Chaudhuri, U. 2013. "Chronic Myeloid Leukaemia in Children and Adolescents: A Single Centre Experience from Eastern India.” South Asian Journl of Cancer 2 (4): 260-4.

[16] Belgaumi, A. F., Al-Shehri, A., Ayas, M., Al-Mahr, M., Al-Seraihy, A., and Al-Ahmari, A. 2010. "Clinical 
Characteristics and Treatment Outcome of Pediatric Patients with Chronic Myeloid Leukemia.” Haematologica 95 (7): 1211-5.

[17] Millot, F., Baruchel, A., Guilhot, J., Petit, A., Leblanc, T., Bertrand, Y., et al. 2011. "Imatinib Is Effective in Children with Previously Untreated Chronic Myelogenous Leukemia in Early Chronic Phase: Results of the French National Phase IV Trial.” J Clin Oncol. 29 (20): 2827-32. Accessed May 27, 2019. http://ascopubs.org/doi/10.1200/JCO.2010.32.7114.

[18] Kerkelä, R., Grazette, L., Yacobi, R., Iliescu, C., Patten, R., Beahm, C., et al. 2006. "Cardiotoxicity of the Cancer Therapeutic Agent Imatinib Mesylate.” Nat Med. 12 (8):
908-16. Accessed May 27, 2019. http://www.ncbi.nlm.nih.gov/pubmed/16862153.

[19] Andolina, J. R., Neudorf, S. M., and Corey, S. J. 2012. "How I Treat Childhood CML Jeffrey." Blood 119 (8): 1821-30. Accessed May 27, 2019. http://www.ncbi.nlm.nih.gov/pubmed/1985699.

[20] Babatunde, T., Ogun, G., Brown, B., Akang, E., and Aken, O. 2014. "Pattern of Childhood Leukaemia in University College Hospital Ibadan.” African J Med Sci. 43 (2): 135-8.

[21] Ngaeje, M., Chinemere, F., Magness, A., and Markani, A. 2016. "Case of a Child with CML Presenting with Vision and Hering Loss.” J Blood Disord Transfus. 7 (1): 33. 